\title{
From Desktop to Tabletop: Migrating the User Interface of AgilePlanner
}

\author{
Xin Wang, Yaser Ghanam, and Frank Maurer \\ Department of Computer Science, University of Calgary, Canada \\ \{xin, yghanam, maurer\} @cpsc. ucalgary.ca
}

\begin{abstract}
Digital tabletops are emerging interactive systems that support group collaborations. To utilize digital tabletops for agile planning meetings, we migrated a desktop based planning tool - AgilePlanner to a digital tabletop. This paper reports on challenges of the migration and illustrates differences between user interactions on a digital tabletop and on a desktop. Moreover, lessons and experiences learnt from our design process are highlighted to facilitate future tabletop application design.
\end{abstract}

Keywords: desktop computer, digital tabletop, user interface design, agile planning tool.

\section{Introduction}

Desktop computers have dominated computer applications for several years. Many activities such as browsing websites and online shopping involve interactions between a desktop system and a computer user. Typical desktop computers are characterized by three basic facts: a vertical display, a single keyboard \& mouse and a relatively small screen. Personal computers are called "personal" because they primarily only support interactions of a single individual with the computer.

However, today's business looks at supporting an increasing number of group interactions. A typical example is an agile planning meeting which requires the software developers, project managers and customers working together as a group to derive release and iteration plans for the next development step. To support agile planning meetings, we had developed a desktop-based application - AgilePlanner [1]. However, some usability problems were observed:

- It is difficult for collocated meeting attendees to share the AgilePlanner interface since it was limited by the small screen size of personal computers. Some agile teams use projectors to get a large display. However, the screen resolution is still limited. Also, the location of the projection screen focuses their attention to the screen and face-to-face interactions are reduced.

- The single input devices impacted group interactions. To use AgilePlanner, meeting participants have to share the mouse and keyboard. That was commented "unnatural", "inflexible" and "annoying". It also slowed down interactions when compared to index cards and pen. 
To overcome usability problems of the desktop AgilePlanner, we started to use a digital tabletop with a large, horizontal and multi-touch screen (see Figure 1). In a tabletop-based meeting, participants could sit or stand around the table, communicate with each other and use their finger touches to interact with the tabletop. User interaction with the tabletop is more intuitive than that with the desktop [2].

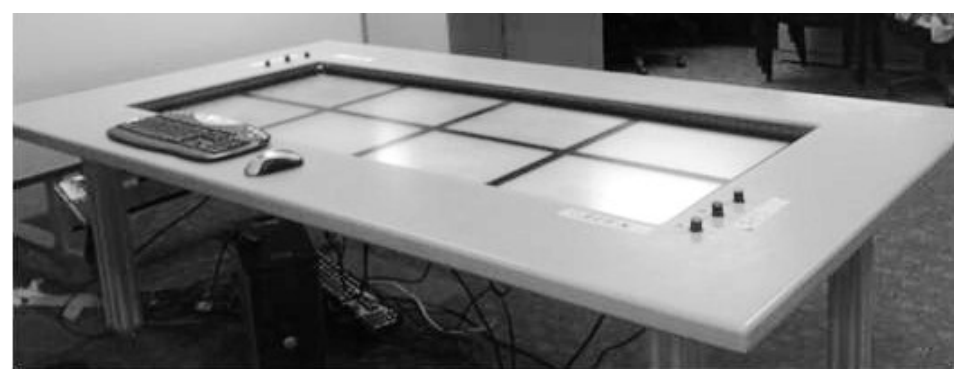

Fig. 1. The $183 \mathrm{~cm} \times 122 \mathrm{~cm}$ digital tabletop with 10 mega pixels output resolutions

Then we migrated our agile planning tool to a digital tabletop. The migration process kept the core functions of desktop AgilePlanner, initially utilized the existing user interface design, and then integrated tabletop usability features. Moreover, lessons and experiences learnt from the migration provide insights into the interaction design of tabletop applications.

This paper is organized as follows: Section 2 discusses related work on user interface migrations. Section 3 illustrates the 4-phase process for migrating AgilePlanner. Section 4 lists the key findings from the migration process. A conclusion is drawn in Section 5 .

\section{Related Works}

There is some research on the migration of user interfaces but none specifically looking into migrating UIs to digital tabletops. Bandelloni et al presented a new environment to support the migration of Web based user interface through different modalities [3]. Mori et al migrated a user interface between Digital TV and mobile devices [4]. Other studies of user interface migrations involve the automatic translation and generation of different Web based user interface languages.

Web based applications are basically running on a similar hardware platform as personal computers, PDAs and cell phones. The main difference is screen resolution and the use of HTML instead of native widget libraries. The interactions for user interfaces are similar. However, a digital tabletop has different user interaction features:

- Tabletop supports touch and gesture recognition. Using a fingertip instead of a mouse reduces accuracy and makes precise selections difficult.

- The relatively large physical size screen combined with touch input makes reaching objects on the screen difficult as human arms have a limited length. 
- The orientation independent display makes it difficult to read text for some meeting participants as it is upside down from their perspective.

- People are aware and often take ownership of parts of the table surface. "Territoriality" in the tabletop workspace was studied in [5].

- Studies of multiple input approaches (mouse, keyboard, finger and pen) for tabletop applications are required [6].

However, experiences about migrating desktop software applications to the digital tabletop are - to the best of our knowledge - not yet reported. Our project, in particular, explored a concrete migration of a desktop based application to tabletop devices.

\section{The Migration Process}

In this section, we will show a migration process that converts the desktop AgilePlanner to the tabletop environment. The migration was organized in four basic phases: analyzing the desktop AgilePlanner, evaluating AgilePlanner on the digital tabletop, redesigning the AgilePlanner UI and continued improvement of the redesign. Usability evaluations are conducted often to validate changes for AgilePlanner. The evaluations included task centered walkthroughs, questionnaire surveys, field studies and interviews with AgilePlanner users.

\subsection{Phase 1: Analyzing Desktop Based AgilePlanner}

Desktop AgilePlanner is a groupware tool for agile planning meetings. Compared to a traditional agile planning meeting that uses paper index cards and a table, the tool provides a flexible, computer-aided, distributed environment. It is easy to operate, e.g. create, modify and resize electronic index cards. Moreover, the meeting results, such as the card contents and the card order that represent task significance, are saved and can be recovered for the next planning meeting.

The user interface design of AgilePlanner (see Figure 2) provides basic functionalities. A large, scrollable workspace is provided to organize the index cards. A vertical legend bar shows card icons that can be dragged to create index cards on the workspace. The horizontal menu makes basic functions such as server connection and card print available to the user. The main user interactions include creating, deleting, resizing and moving index cards. A keyboard is used to conduct card editing and modifications.

Desktop AgilePlanner is designed for the typical personal computer featuring a small, vertical display with single keyboard \& mouse control that supports individual interactions. But in a multi-user collocated team, some limitations are observable. For example, suppose there is an 11-person team which consisted of 2 collocated groups (one in Vancouver with 5 people and the other in Calgary with 6 people). To conduct an 11-person meeting which is distributed between Vancouver and Calgary but collocated inside the two groups, the attendees have to use their individual computers and thus, face to face communication of the collocated teams is changed. A possible alternative is using projectors to enlarge and project the AgilePlanner interface to a shared screen. However, the projected screen becomes the focus of attention of onsite team members, thus reducing the effectiveness of collocated communication. Moreover, 
some natural behaviors in traditional agile meetings, such as passing card among several participants, rotating cards and concurrently operating cards, are not supported by AgilePlanner or similar tools as a single user is controlling the mouse \& keyboard.

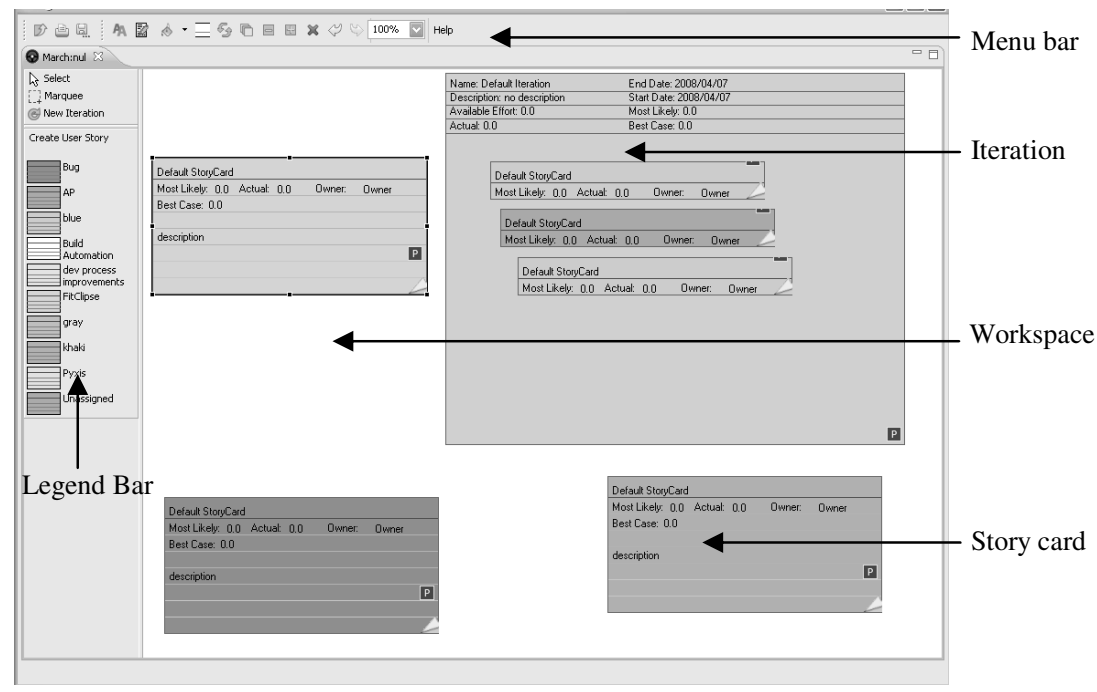

Fig. 2. User interface of desktop AgilePlanner

\subsection{Phase 2: Evaluating AgilePlanner on the Tabletop}

In this phase, we conducted a usability evaluation of the desktop AgilePlanner tool after deploying it on a digital tabletop. The goal of this evaluation is to highlight the differences of desktop and tabletop systems, in particular, the size of screens, the horizontal versus vertical display, as well as the single versus concurrent users. The evaluation was designed as a task-centered walkthrough that employed 6 testers. During the evaluation, the testers were required to complete some sample tasks using the desktop AgilePlanner which was running on the digital tabletop. We discovered several usability problems through this study. Design guidelines for a redesigned tabletop-based AgilePlanner were developed based on an analysis of the problems. The following subsections illustrate the basic differences of desktops and tabletops.

\section{Vertical vs. horizontal display}

A typical desktop computer often provides a vertical screen which only requires a topdown (vertical) orientation: there is a defined upper edge and a defined lower edge. Desktop applications are designed with this in mind. Using AgilePlanner as an example, story cards are all placed vertically. However, horizontal displays are orientation independent and require rotating cards to show them to people on the other side of the table.

\section{Single vs. concurrent interaction}

Desktop computers are based on single mouse-keyboard interactions. The desktop AgilePlanner can only respond to one mouse-keyboard action at the same time. 
However, a real collocated planning meeting often involves several participants who operate story cards simultaneously. The lack of simultaneous interactions reduced the agile developers' motivation to use AgilePlanner. For example, if a meeting participant wants to edit a story card, she must negotiate with other participants to get the keyboard and mouse control. Moreover while she was editing story cards, other participants could not input information till the completion of her operation. Our testers noticed the inconvenience caused by the single mouse-keyboard interaction. They commented it "unnatural" and "interrupt communications".

However, the digital tabletop support multi-touch input. Meeting attendees are able to use their fingers to operate the story cards simultaneously. For example, two or more meeting attendees could use their fingertips to write text, and their handwriting strokes would be kept and converted to text.

\section{Small vs. large display}

Several usability problems from the evaluation are related to the different screen sizes of desktops and tabletops. For instance, a popup dialog box is a common interaction component. AgilePlanner often shows these at the center of the computer screen. However, our physical tabletop surface is at least 8 times larger than a normal screen of a desktop computer. Thus, the pop up position of the dialog box might be out of reach of a user sitting at the end of the table. One of our study subjects mentioned that he was often stopped by the pop-up dialog box. He commented that "finding and clicking the pop-up dialog box are both annoying".

\section{Recommendations}

The following guidelines for redesigning the AgilePlanner user interface were derived from our study:

1. UI components of AgilePlanner should be moveable and rotatable.

2. Use gesture recognition for user interactions and avoid traditional menus.

3. Use handwriting instead of the keyboard to input text.

4. Consider concurrent interaction while designing the UI.

5. The size of widgets of the tabletop AgilePlanner should be large enough to facilitate touch input.

6. Avoid using pop up dialog boxes and other similar components.

7. Since the size of tabletops varies a lot, it is necessary to make the application interface scalable.

\subsection{Phase 3: Redesigning the AgilePlanner User Interface}

Based on the guidelines from the phase 2, AgilePlanner was redesigned. We found that Microsoft WPF (Windows Presentation Foundation) better supported tabletops than Java SWT (the framework underlying the desktop version of AgilePlanner). WPF provides a sound basis for tabletop applications. UI components of WPF can easily be transformed in size, position and angularity. Handwriting, gesture and voice recognition engines are provided by the WPF environment.

We abandoned the traditional WIMP (window, icon, menu, pointer device) approach in the desktop applications. The menus and legend bars are integrated into a control palette which can be moved and rotated on the table surface to allow access from any seat around the table. A rotation and translation (RNT) algorithm was 
implemented to facilitate the movement of the story cards, iterations and the control palette. Moreover, we implemented handwriting recognition to translate strokes into text. The original handwritings are kept and displayed on the story card surface. The control flow of AgilePlanner is also simplified. Instead of clicking buttons with a mouse, users can make simple gestures to complete the card operations. Figure 3 lists some gestures defined for card operations. A story card is created by a "ChevronDown" gesture. The "Square" gesture creates iteration. To delete an index card, a "ScratchOut" gesture is required to exceed the whole card boarder. Our evaluation indicates that gestures are flexible, learnable and easy to use. Moreover, pop up dialog boxes are replaced by customized windows which have a large size and can be moved on the table surface. System warnings are displayed on the control palette. The new user interface of tabletop AgilePlanner is shown on the Figure 4:

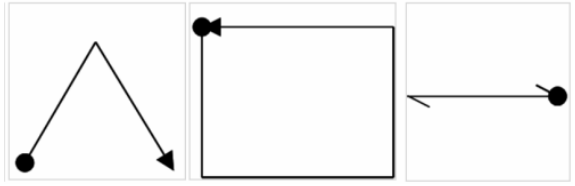

(a)

(b)

(c)

Fig. 3. Gestures commands for Tabletop based AgilePlanner (a) create story card, (b) create iteration, (c) delete card

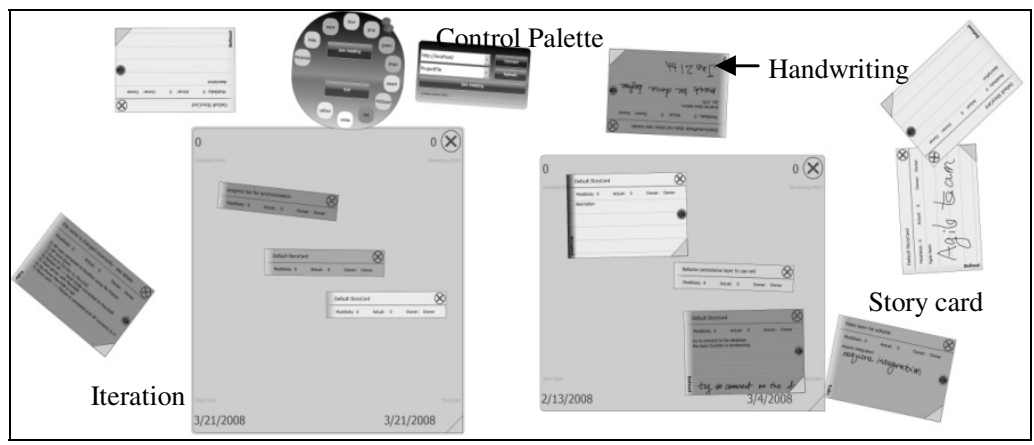

Fig. 4. User interface of tabletop based Agile Planner

To validate the new UI, we conducted a pilot evaluation which included 14 subjects. They were asked to complete sample tasks and filled out a questionnaire afterwards. Unstructured interviews were used to collect the testers' comments. The results of the pilot evaluation showed the users' satisfactions with the new UI and interaction design. Most of the testers felt comfortable when using the tabletop based AgilePlanner. Some negative comments and usability issues were arisen. For example, some testers suggested the "deleting card" gesture (see Figure 4(d)) was confusing while others commented the handwriting "cool but sometimes unreadable". 


\subsection{Phase 4: Continued Improvement}

We continue development to solve the usability problems found in Phase 3 . We analyzed the card deletion gestures and found out that most of testers were not aware the deletion gesture should go through the whole card. Moreover, when the card is very large, drawing the deletion gesture will be difficult. As an alternative approach, a card deletion button is placed at the right corner of an index card. We also studied the problems of handwriting readability and found out that the fingertips of testers were very thick so that it was difficult to draw tiny ink strokes accurately. Moreover, some testers mentioned that using their fingers to write on the table surface was unnatural because, while writing with fingers, their fists were not allowed to touch the table.

We reevaluated the system after making corresponding changes. 9 testers showed their satisfactions (see Figure 5) to the functionalities provided by AgilePlanner. But the usability of handwriting on tabletop still requires the improvements.

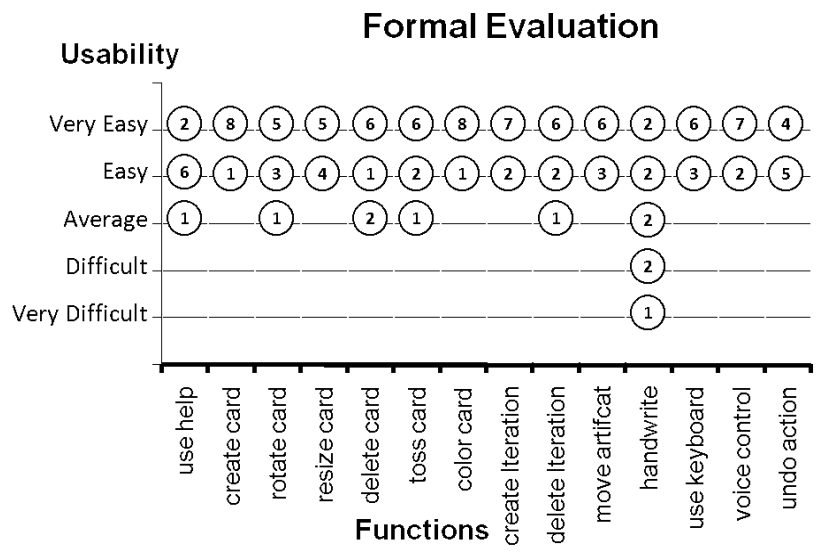

Fig. 5. Formal evaluation result

\section{Discussion}

From the migration process, we gathered experiences that can help other developers of tabletop applications.

The horizontal display brings the orientation independence to the user interface. Thus, rotating the UI elements of AgilePlanner became necessary. The physical screen sizes of tabletops vary a lot. On a large tabletop (like ours), menu bars or popup windows with fixed positions and small sizes are hard to find and click. Thus, the UI components need to be scalable to fit different tabletop surfaces.

Tabletop devices are touch sensitive. Tabletop developers can make use of the touch recognition to support a gesture and handwriting. However, "unnatural" gesture definitions might cause severe confusions. Thus, although a gesture and finger interaction is often more flexible than the mouse actions, its implementation must be based on careful design and evaluation. 


\section{Conclusion}

The digital tabletop is gaining its popularity as an emerging technology to support group activities. However, not many tabletop based applications for real end users exist. We migrated the desktop AgilePlanner to the tabletop environments to gain a better understanding of issues involved in application engineering for tabletop-based software systems.

The migration had 4 phases. The first phase helped to understand the UI and interaction design of the source application. In the second phase, we evaluated the existing application in the new environment. A new tabletop AgilePlanner was designed in Phase3. The focus of the redesign was on utilizing the capabilities of tabletops and improving the application usability. In Phase 4, continued improvements were made to fulfill new user requirements or solve usability problems.

We discussed the differences of the desktop and tabletop interactive systems. Some UI design experiences provide rough guidelines to help tabletop application developers in migrating other applications.

\section{References}

1. Liu, L., Erdogmus, H., Maurer, F.: An environment for collaborative phase planning. In: Proceedings of Agile Conference, pp. 80-89. ACM, New York (2005)

2. Morgan, R., Walny, J., Kolenda, H., Ginez, E., Maurer, F.: Using Horizontal Displays for Distributed \& Collocated Agile Planning. In: Concas, G., Damiani, E., Scotto, M., Succi, G. (eds.) XP 2007. LNCS, vol. 4536, pp. 38-45. Springer, Heidelberg (2007)

3. Bandelloni, R., Paternò, F., Santoro, C., Scorcia, A.: Web User Interface Migration through Different Modalities with Dynamic Device Discovery. In: Proceedings AEWSE 2007, Como, pp. 58-72 (2007)

4. Mori, G., Paternò, F., Santoro, C., Sansone, S.: Migrating the User Interface between the Digital TV and Mobile Devices. In: Proceedings Interacive TV: A shared experience, Amsterdam, Holland, pp. 73-77 (2007)

5. Scott, S.D.: Sheelagh M., C.T.I. Kori: Territoriality in collaborative tabletop workspaces Tabletop design. In: Proceedings of ACM Conference on Computer-Supported Cooperative Work, pp. 294-303. ACM Press, New York (2006)

6. Tse, E., Shen, C., Greenberg, S., Forlines, C.: Enabling interaction with single user applications through speech and gestures on a multi-user tabletop. In: Proceedings of the conference on Advanced visual interfaces, pp. 336-343. ACM Press, New York (2006) 